\title{
Intermittent activity of radio sources. Accretion instabilities and jet precession.
}

\author{
M. Kunert-Bajraszewska ${ }^{1}$, A. Janiuk ${ }^{2}$, A. Siemiginowska ${ }^{3}$ \\ and M. Gawroński ${ }^{1}$ \\ ${ }^{1}$ Toruń Centre for Astronomy, N. Copernicus University, Gagarina 11, 87-100 Toruń, Poland \\ email: magda@astro.uni.torun.pl \\ ${ }^{2}$ Center for Theoretical Physics, PAN, Al.Lotnikow 32/46, 02-668 Warsaw, Poland \\ ${ }^{3}$ Harvard Smithsonian Center for Astrophysics, 60 Garden St, Cambridge, MA 02138
}

\begin{abstract}
We consider the radiation pressure instability operating on short timescales $\left(10^{3}-10^{6}\right.$ years) in the accretion disk around a supermassive black hole as the origin of the intermittent activity of radio sources. We test whether this instability can be responsible for short ages $\left(<10^{4}\right.$ years) of Compact Steep Spectrum sources measured by hot spots propagation velocities in VLBI observations and statistical overabundance of Gigahertz Peaked Spectrum sources. The implied timescales are consistent with the observed ages of the sources. We aslo discuss possible implications of the intermittent activity on the complex morphology of radio sources, such as the quasar $1045+352$, dominated by a knotty jet showing several bends. It is possible that we are whitnessing an ongoing jet precession in this source due to internal instabilities within the jet flow.
\end{abstract}

Keywords. physical data and processes: accretion, galaxies: evolution, quasars: individual $(1045+352)$

\section{Introduction}

The compact radio sources consist of two population of objects: the gigahertz-peaked spectrum (GPS) and compact steep spectrum (CSS) sources which are considered to be young and evolve into large radio objects, during their lifetimes (O'Dea, 1998). However, it has already been pointed out by some authors (Gugliucci et al. 2005, Kunert-Bajraszewska et al. 2006) that there exists a group of GPS/CSS sources that will never evolve to become large scale objects, at least in a given cycle of activity if it is recurrent. These sources can be named short-lived radio objects.

We associated the existence of short-lived compact radio sources with the intermittent activity of the central engine caused by a radiation pressure instability within an accretion disk, which we briefly mention below.

\section{Discussion and Results}

According to the accretion disk instability model, for a given black hole mass, the larger the mean accretion rate, the longer the duration of a cycle episode, both in hot and cold states. In addition the viscosity parameter affects the results and for smaller viscosity, the cycle duration is longer. This relation was calibrated by Czerny et al. (2009) using a grid of models for various black hole masses and Eddington ratios. The outbursts are associated with the ejections of radio jets. The jets are then turned-off between the outbursts and each radio structure will represent a new outburst. In case of an apparently young, compact source we can suspect that in fact it is an old, reactivated object, in which 


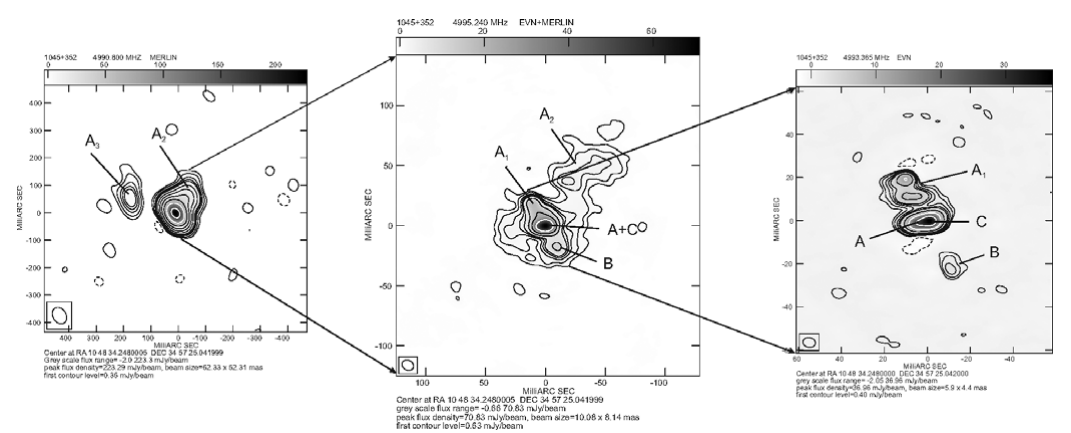

Figure 1. Radio images of $1045+352$ at $5 \mathrm{GHz}$ made with (from left): MERLIN, EVN+MERLIN, EVN. Contours increase by a factor 2 and the first contour level corresponds to $3 \sigma$. Indications: $\mathrm{C}$ - radio core, $\mathrm{A}-A_{3}$ - radio jet, $\mathrm{B}$ - probable counter-jet.

the vast radio structures have already faded away and are not visible. We have applied i.a. the accretion disk instability model and jet precession model to explain the potential reactivation of the $1045+352$ core (Kunert-Bajraszewska et al. 2010).

$1045+352$ is a CSS object and a HiBAL quasar at a medium redshift. Its linear size $(\sim 4 \mathrm{kpc})$ indcate it is a young object in the early phase of quasar evolution. The radio morphology of $1045+352$ is dominated by the strong radio jet resolved into many subcomponents and changing the orientation during propagation in the central regions of the host galaxy. As a consequence we observe at least three phases of jet activity indicate different directions of the jet outflow: components $A_{2}-A_{3}$ as the oldest one, structure $A_{1}$-B as the younger one, and the jet $\mathrm{A}$ as the current activity direction (Fig.1).

The results of the applied accretion disk instability model (Janiuk et al. 2002, Czerny et al. 2009) is not in agreement with the 1045+352 structure and size: (a) the calculated duration of the activity phase of the quasar is too short to enable the source grow to the observed size, (b) it cannot reproduce misalignment between the young and old radio structures. Such misalignment could be the result of the changed direction of the jet axis between the activity episodes - precession. In the case of $1045+352$ we considered the precession of the innermost accretion disk due to internal instabilities within the accretion flow (Janiuk et al. 2008). This precession model reproduced well the observed complex structure of $1045+352$. However, as discussed by Czerny et al. (2009), the mechanism of the accretion disk instability can be considered as the one that could explain the apparent statistical excess of the compact radio sources with respect to the galaxies with extended radio structures.

\section{Acknowledgements}

This work was supported by the Polish Ministry of Science and Higher Education under grant N N203 303635.

\section{References}

Czerny, B., Siemiginowska, A., Janiuk, A., Nikiel-Wroczyński, B., \& Stawarz, Ł., 2009, ApJ, 698,840

Gugliucci, N. E., Taylor, G. B., Peck, A. B., \& Giroletti, M., 2005, ApJ, 622, 136

Janiuk A., Czerny B., \& Siemiginowska A., 2002, ApJ, 576, 908

Janiuk A., Proga D., \& Kurosawa R., 2008, ApJ, 681, 58

Kunert-Bajraszewska, M., Marecki, A., \& Thomasson, P., 2006, A $\& A$, 450, 945

Kunert-Bajraszewska, M., Janiuk, A., Gawroński, M. P., \& Siemiginowska, A., 2010, ApJ, 718, 1345

O’Dea, C. P., 1998, PASP, 110, 493 